\title{
Application of Genetic Algorithm in Common Optimization Problems
}

\author{
Nika Topuria*, Omar Kikvidze \\ Department of Technical Engineering, Akaki Tsereteli State University, Georgia \\ * Corresponding author email: nicolas1213@gmail.com
}

Received: 07 June 2019 / Revised: 26 June 2019 / Accepted: 06 July 2019 / Published: 21 July 2019

\begin{abstract}
Use of non-deterministic algorithms for solving multi-variable optimization problems is widely used nowadays. Genetic Algorithm belongs to a group of stochastic biomimicry algorithms, it allows us to achieve optimal or near-optimal results in large optimization problems in exceptionally short time (compared to standard optimization methods). Major advantage of Genetic Algorithm is the ability to fuse genes, to mutate and do selection based on fitness parameter. These methods protect us from being trapped in local optima (Most of deterministic algorithms are prone to getting stuck on local optima). In this paper we experimentally show the upper hand of Genetic Algorithms compared to other traditional optimization methods by solving complex optimization problem.
\end{abstract}

Keywords: Oncology, Optimization, Genetic, Non-Deterministic, Algorithm

\section{Introduction}

Modern medical researches involve more math and calculations than ever, including the optimization of large experimental datasets and complex bio-chemical simulations. Modern radiation therapy heavily uses optimization algorithms [1]-[3] to achieve best possible tumor irradiation while protecting the surrounding healthy tissue. To achieve this, the optimization algorithm must find best possible irradiation angles [4] and dose fluence maps based on multiple constraints set by a medical physicist. The problem here is a complexity of the optimization area (irregular tumor shape, irregular patient surface, multiple healthy organs surrounding the tumor) and the limited time to make an optimal treatment/irradiation plan.

Even though there exists a software for automatic irradiation angle selection, but most of the hospitals can't afford to have it installed on their workstations. There are several significant reasons for that, for example, BAO algorithms are quite expensive and most of small hospitals can't afford to have it on their workstation(s). In addition to being expensive, the runtime of a common optimization process is painfully slow [5], requires pretty powerful computer and usually takes most of the processing power of the workstation making it less usable for other tasks while optimization is running. While it's hard to get the best possible plan from the optimizer in reasonable time, it's possible to tradeoff $q$ bit of the plan quality for the time required for calculations. All of above-mentioned problems can be resolved purely by proper software and optimization algorithms. It's well known [6][7][8][9] that most of the large scale, multi variable, hard constrained minimization problems can be hard to solve (in time) for traditional deterministic optimization algorithms, while stochastic approach can give reasonably optimal solutions in marginally short time.

In this paper we employ a certain type of stochastic algorithms - Genetic Algorithm [10] to solve multi variable, heavily constrained IMRT (Intensity Modulated Radiation Therapy) beam angle optimization problem. 
Application of Genetic Algorithm in Common Optimization Problems

\section{Materials and Methods}

Genetic algorithm has been around for quite a while now (Initially described by John Holland in 1960s [11]) and is frequently used for modern-day optimization problems. There exist several types of Genetic Algorithms (GA in future references). This kind of algorithms must have a set of the following five components to work properly:

- A genetic representation for potential solutions to the problem.

- A way to create an initial population of potential solutions.

- An evaluation function that plays the role of the environment, rating solutions in terms of their fitness to the environment.

- Genetic operators that alter individuals for the composition of children.

- Values for various parameters that the Genetic Algorithm uses (Population size, probabilities of applying genetic operators, etc.).

As mentioned above Genetic algorithm uses population-based approach to the optimization problem. This means that there are several virtual entities or so called "seekers" in every GA instance, each representing a potential solution to the task (in our case - set of beam angles). During each optimization step, the quality of each seeker is evaluated using a Fitness function - calculating how good they are relative to the problem domain. After each evaluation round, comes Selection, which is a way for GA to move towards better search area. The working principle is somehow like natural selection - fittest individuals (seekers with better scores on evaluation round) have highest chances to survive for next iteration. This way worst possible angle sets are eliminated from the search area, resulting in simplification of the search task for remaining seekers. However, if we never create new individuals our optimization process will end prematurely - giving unacceptably bad results, therefore we have an artificial reproduction system for the seekers - a Crossover. This is a procedure of combining two individual "genes" (each 'gene' is an angle in our case) to produce offspring which inherits best characteristics of their parents. Simplest example of crossover is single point crossover which is shown bellow:

Parent 1 DNA: 01010110

Parent 2 DNA; 11100001

Crossover points: 3

Child 1 DNA: 01000001

Child 2 DNA: 11110110

It's well known that deterministic algorithms are usually prone to being entrapped in a local extreme point of the optimization curve [12]. This can be avoided by the introduction of random gene alteration in one or two seekers, also called Mutation - is a genetic operator that plays major and distinctive role in the performance of GA. It prevents the population from stagnating at any local optima, technically it means that you take one or more random chromosomes (angles) from DNA (set of angles) string and change it. The optimization is finished when no measurable improvements are observed for predefined number of generations.

In order to show effectiveness of GA for IMRT beam angle optimization problem, we have created treatment plans for three real-life treatment areas, three for each, using our GA based solution, traditional equispaced beam setups and Varian BAO commercial software.

MATLAB has been chosen as a programing language. The basis of the project is an open source framework MatRad [13]. It contains all the required tools to do the treatment planning including ray tracing and inverse planning functionality. Source code developed by us during the research will be added to the MatRad system with the authors consent.

\section{Calculations and Results}

The algorithm has been tested on 3 clinical cases: Brain tumor, Skin tumor (on the nose), Lung tumor. For each case an IMRT (Intensity Modulated Radiation Therapy) plan has been created using genetic algorithm. Resulting angles has been imported to Varian Eclipse treatment planning system for final dose distribution calculation. The optimization was done on Dell Precision T5600 with 2x - E5-2620 and 32Gb Ram. 
Topuria et al., Int. Ann. Sci.; Vol. 8, Issue 1, pp: 17-21, 2020

In order to eliminate unwanted biasing, optimization parameters for both our GA-BAO implementation and Varian BAO framework were exactly the same. GA-BAO gave most notable improvements compared to other methods in Brain tumor case. Figure 1 shows the comparison of Dose Volume Histograms from genetic based optimizer and Varian BAO. Overall evaluation of the comparison shows decreased healthy tissue doses with better target coverage and homogeneous dose distribution for GA. Optimization time -86 minutes.
Lung tumor - In this case Varian BAO gave clearly unacceptable field set, having some of the fields entering body from the direction of healthy lung. Therefore, for this case only man-made and GA$\mathrm{BAO}$ plans were evaluated. Even though generally for lung cancers experience based manual planning is a preferred way to go, our methodology gave better overall plan quality and dose distribution to the target (Figure 2). Also, doses for both lungs and heart is marginally better while maintaining good target coverage. Optimization time - 134 minutes.

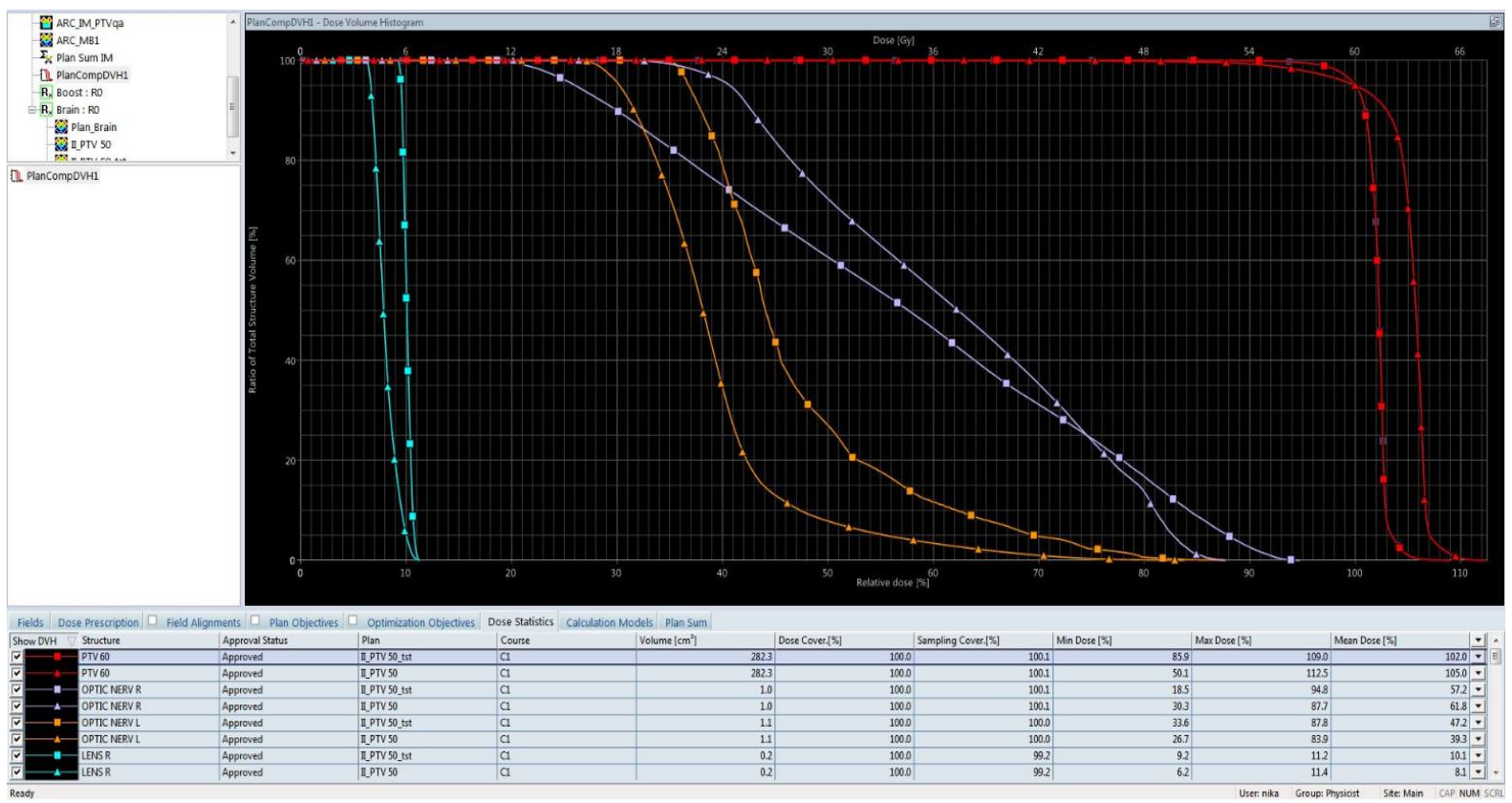

Figure 1: DVH (Dose Volume Histogram) comparison for brain tumor plan, GA-BAO vs. Varian BAO

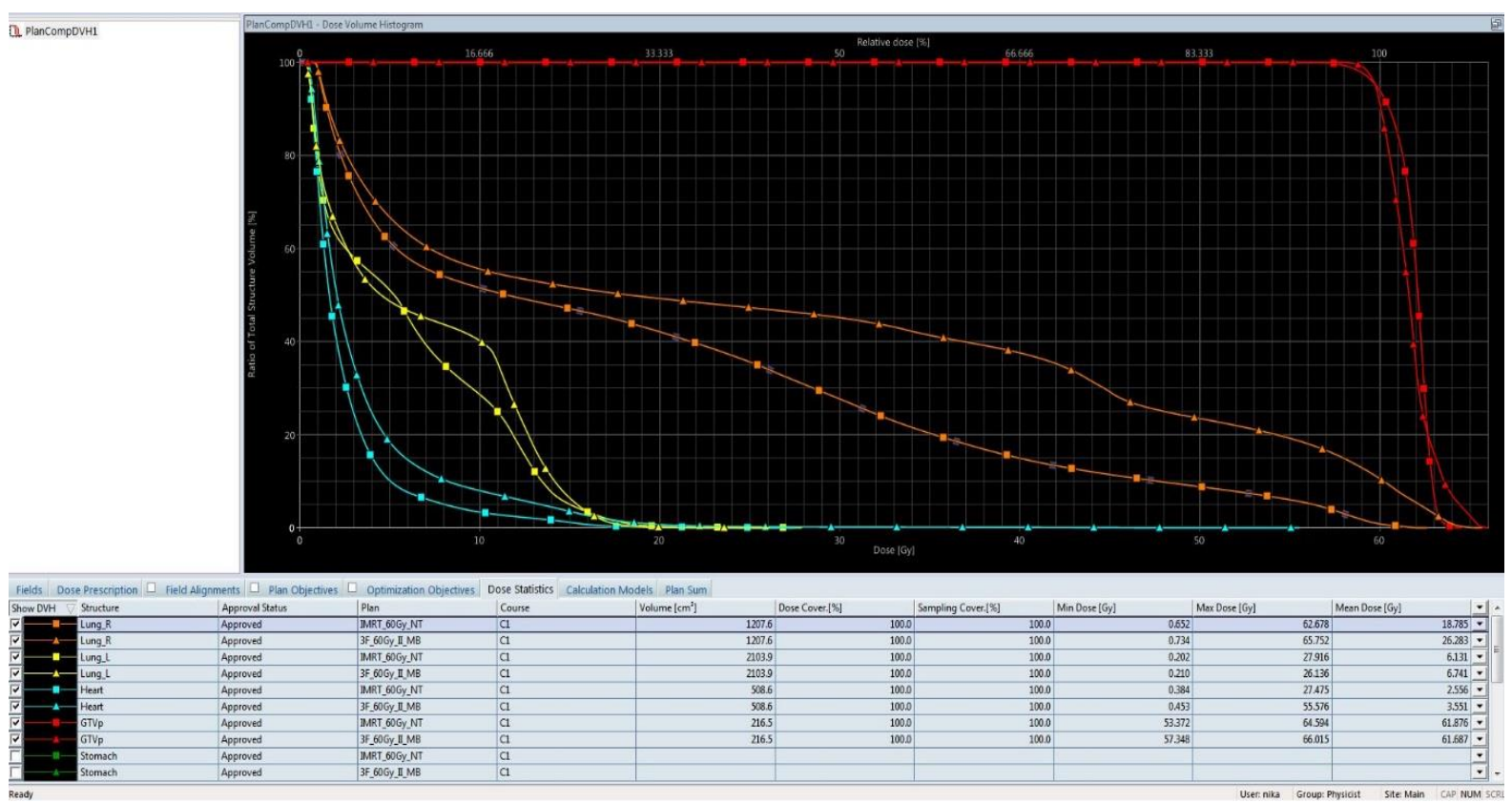

Figure 2: DVH (Dose Volume Histogram) comparison of human-made and GA-BAO plans 


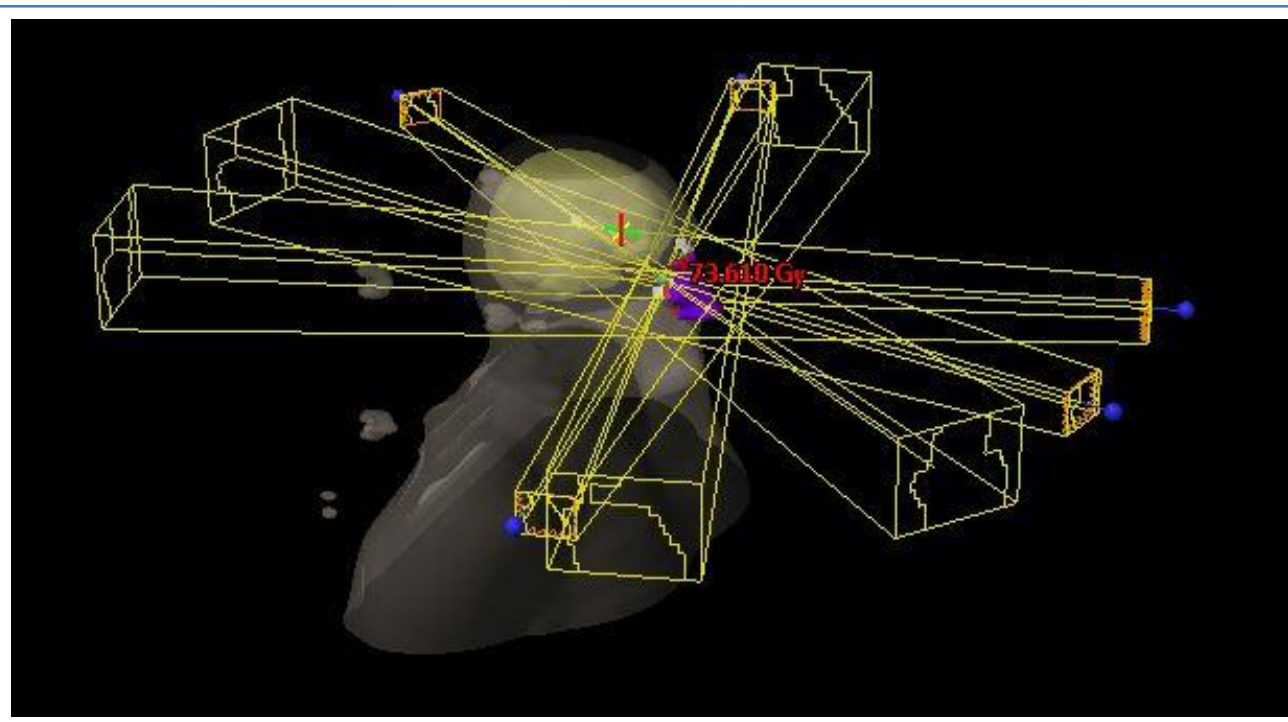

Figure 3: Coplanar plan field alignment around the patient created by GA-BAO

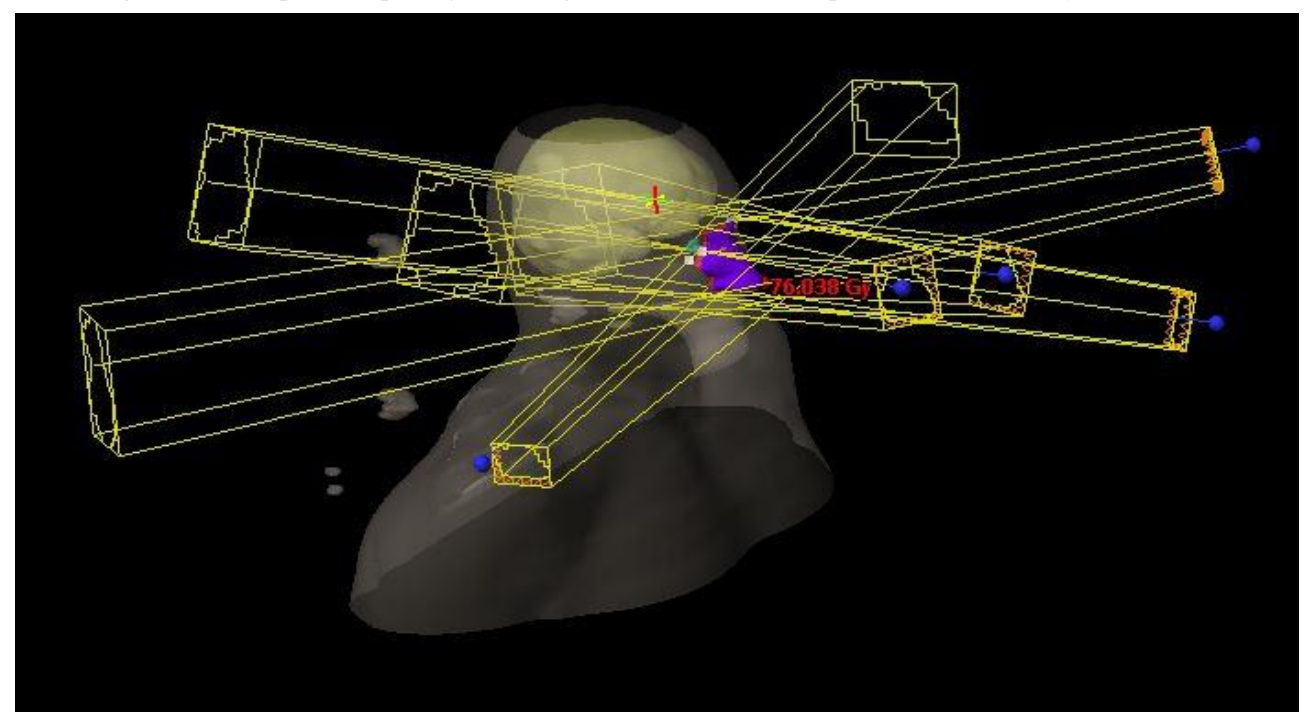

Figure 4: Non-coplanar plan field alignment around the patient created by the expert

The only case where GA-BAO was beaten was the Skin tumor. In this case our plan was slightly better than the one created by Varian BAO but was slightly worse than the one created by an expert, however the difference between these three were insignificant. The reason behind expert-made plan being the best is that other plans consisted of only coplanar fields (Figure 3), while the expert used non-coplanar field alignment (Figure 4), increasing dose modulation and healthy tissue sparing even further.

To summarize the results GA-BAO approach definitely has potential to replace currently used $\mathrm{BAO}$ techniques and further improve treatment plan quality. GA algorithm proved to be versatile tool for planning, since it could easily eliminate obviously bad angles from the beginning steps of calculations and never got stuck in local extreme point of the optimization curve. As it is right now, the main weakness of our implementation is its inability to use non-coplanar fields, however this feature will be added in our future works. Regarding optimization speed: while GA-BAO was mostly faster than Varian BAO, its speed can be further improved by better parallelization and code tweaking.

\section{Conclusions}

This paper describes the nature of Genetic algorithm, its structure and one specific application case. We present a comparison of three IMRT beam angle selection methods in 
Topuria et al., Int. Ann. Sci.; Vol. 8, Issue 1, pp: 17-21, 2020

terms of resulting treatment plan quality, showing that the application of genetic algorithm for multi-variable, hard constrained, large scale optimization tasks can be a good solution to the typical technical problems of standard deterministic approaches. Calculation results showed that, in every case, except one, GA-BAO got better normal tissue sparing and better dose homogeneity compared to plans created by human expert or Varian BAO algorithm. In general, the versatility of evolutionary algorithms makes GA a feasible tool for treatment planning for mostly all treatment locations. Early testing indicates that genetic algorithm in radiation therapy has quite promising future and points out important areas where improvements can be made. Further development can decrease required time for optimization (Using GPU for ray tracing and large matrix manipulations) [15] and pre-optimization filtration of obviously infeasible field angles can shrink the search area, while added non-coplanar mode will increase resulting plan quality.

\section{Competing Interests}

The authors declared that no conflict of interest exist in publishing this article.

\section{How to Cite this Article:}

N. Topuria and O. Kikvidze, "Application of Genetic Algorithm in Common Optimization Problems", Int. Ann. Sci., vol. 8, no. 1, pp. 17-21, Jul. 2019. doi:10.21467/ias.8.1.17-21

\section{References}

[1] C. Johansson, G. Evertsson, "Optimizing Genetic Algorithms for Time Critical Problems", Master Thesis, Dep. of Software Engineering and Computer Science, Blekinge Institute of Technology, Sweden, June 2003.

[2] D. Djajaputra, et al. "Algorithm and performance of a clinical IMRT beam-angle optimization system." Physics in medicine and biology, Vol. 48, Issue 19, pp. 3191-212, 2003.

[3] L. Shao, "A survey of beam intensity optimization in IMRT" in Proceedings of the 40th Annual Conference of the Operational Research Society of New Zealand, Department of Engineering Science, University of Auckland, pp. 2-13.

[4] M. Ehrgot, A. Holder, J. Reese. "Beam selection in radiotherapy design.", Linear Algebra and its Applications, Vol. 428, Issues 5-6, pp. 1272-1312, march, 2008.

[5] H. Rocha, J.M. Dias, B.C. Ferreira, M.C. Lopes, "Beam angle optimization for intensity-modulated radiation therapy using a guided pattern search method." Physics in medicine and biology, Vol 58-9, pp.2939-2953, 2013.

[6] H. Yarmand, D. Craft, "Effective Heuristic Cuts for Beam Angle Optimization in Radiation Therapy", Medical Physics, Vol. 40, Issue 6, pp. 387-387, June 2013.

[7] X. Wang, M. Damodaran, "Comparison of Deterministic and Stochastic Optimization Algorithms for Genetic Wing Design Problems", Aerospace Research Central, Vol. 37, Issue 5, pp. 929-932, May 2012.

[8] A.M. Connor, K. Shea, "A Comparison of Semideterministic and Stochastic Search Techniques", Book: Evolutionary Design and Manufacture, Springer, London, pp. 287-298, 2000.

[9] A. Blake, "Comparison of the Efficiency of Deterministic and Stochastic Algorithms for Visual Reconstruction.” IEEE Trans. Pattern Anal. Mach. Intell. Vol. 11, Issue 1, pp. 2-12, January 1989.

[10] A. Beloglazov, "Optimal online deterministic algorithms and adaptive heuristics for energy and performance efficient dynamic consolidation of virtual machines in Cloud data centers", Concurrency and Computation Practice and Experience, Vol. 24, Issue 13, pp. 13971420, September, 2012.

[11] N.M. Razali, J. Geraghty, "Genetic Algorithm Performance with Different Selection Strategies in Solving TSP", Conference: International Conference of Computational Intelligence and Intelligent Systems, January, 2011

[12] M. Mitchell, "Genetic algorithms: An overview", Complexity, Vol, 1, Issue 1, pp. 31-39, May, 2013.

[13] J. A. Purdy, "Three-Dimensional treatment planning and conformal dose delivery" in Advances in Radiation Therapy, B.B. Mittal, J.A. Purdy, K.K. Ang, Springer US, Vol 93, pp. 19-21.

[14] H, Wieser, E. Cisternas, N, Wahl, S. Ulrich, A. Stadler, H. Mescher, L. Müller, T. Klinge, H. Gabrys, L. Burigo, A. Mairani, S. Ecker, B. Ackermann, M. Ellerbrock, K. Parodi, O. Jäkel, and M. Bangert, "Development of the open-source dose calculation and optimization toolkit matRad" Meical. Physics, Vol. 44, Issue 6, pp. 25562568. June, 2017.

[15] N. Hou, F. He, Y. Zhou, Y. Chen, X. Yan "A Parallel Genetic Algorithm With Dispersion Correction for HW/SW Partitioning on Multi-Core CPU and ManyCore GPU," in IEEE Access, Vol. 6, pp. 883-898, 2018.

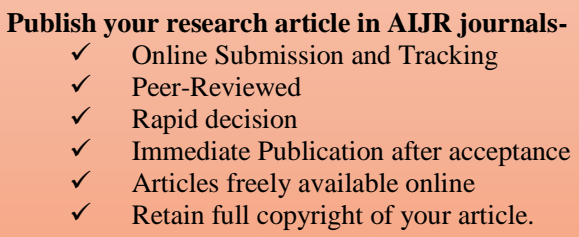

Publish your books with AIJR publisher-

$\checkmark$ Publish with ISBN and DOI.

$\checkmark$ Publish Thesis/Dissertation as Monograph.

$\checkmark$ Publish Book Monograph.

$\checkmark$ Publish Edited Volume/ Book.

$\checkmark$ Publish Conference Proceedings

$\checkmark \quad$ Retain full copyright of your books.

Submit your manuscript at books.aijr.org 